\title{
THE REFLECTION COEFFICIENTS OF METALS FOR THE POLARIZED COMPONENTS OF LIGHT.
}

By R. B. WILSEY.

A NUMBER of observers have determined the reflection coefficients $A$ at normal incidence for various metals. In some cases the agreement between different observers is excellent; in others it is very poor, the discrepancy mounting as high as fourteen per cent. Such a difference is much greater than can be attributed to experimental error. These discrepancies exist not only between those determined polarimetrically and those determined spectrophotometrically, but also between the various polarimetric values. The reasons for these discrepancies as determined by different observers have been summarized by Tate. ${ }^{1}$

Tate having made mirrors of gold and copper by electroplating upon speculum metal, could not obtain surfaces of the same metal whose reflection coefficients agreed within one per cent. All the evidence obtained indicates that the optical properties of a metal surface are not characteristic of the metal alone, but also of the particular surface under investigation. Hence it is to be expected that reflection coefficients determined for different mirrors of the same metal, will, in general, not agree.

It follows then that the observed discrepancies between the reflection coefficients of metals measured photometrically and those calculated from polarimetric measurements do not necessarily invalidate the theory by which reflection coefficients are calculated from measurements of the ellipticity produced in plane polarized light by reflection from the polished surface; since the determinations have always been made, for the two methods, by different observers upon different mirrors.

In order to test more rigidly the validity of the theory of metallic reflection, Tate compared the reflection coefficients at normal incidence obtained by both the polarimetric method and the photometric method upon the same mirrors. His polarimetric measurements were made by a Tool $^{2}$ polarimeter, and his photometric measurements by a Brace

1 Tate, J. T., Phys. Rev., 34, p. 240.

2 Tool, A. Q., Phys. Rev., 3I, p. I, I9Io. 
spectrophotometer provided with a special reflecting device. He tested mirrors of steel, silver, gold, copper and fuchsin, and found excellent agreement between the reflection coefficients obtained polarimetrically and those obtained photometrically. He concluded that the polarimetric determination of metallic reflection coefficients for normal incidence was reliable and found further, that the errors of his measurements showed the polarimetric method was much more accurate than the photometric method.

The purpose of the present investigation was to test more completely the adequacy of the polarimetric method of determining the reflection coefficients of metals, by extending the investigation to the component vibrations parallel and perpendicular to the plane of incidence, at various angles of incidence. The method followed here was the one used by Tate, namely, the practically simultaneous determination of the reflection coefficients of the same mirror by the polarimetric and the photometric methods. The apparatus was the same-the Tool polarimeter and the Brace spectrophotometer with the special reflecting device.

\section{Photometric.}

The only addition here was the introduction of a Glan air nicol $N$ (Figs. I and 2) in the reflecting system to polarize the light incident

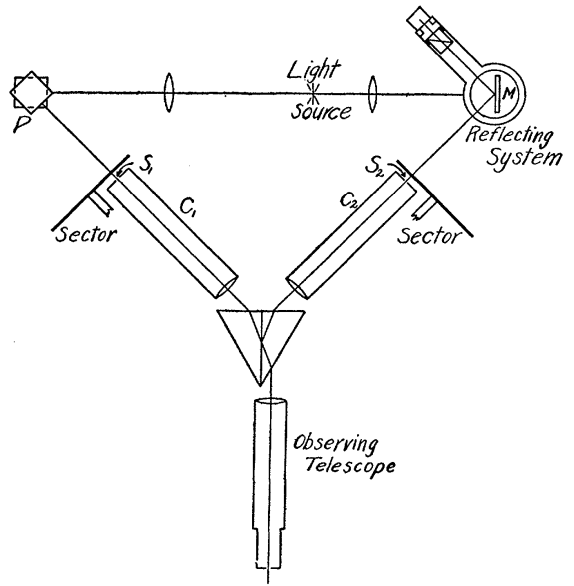

Fig. 1.

upon the mirror. This nicol could be rotated through an angle of $90^{\circ}$ and hence provide light polarized either parallel or perpendicular to the plane of incidence. M'Connel's method ${ }^{1}$ of an auxiliary nicol mounted upon the table of the rotating reflecting system was used for determining the correct positions of the polarizing nicol.

${ }^{1}$ M'Connel, J. C., Phil. Mag. (5), vol. I9, p. 3I7, I 885. 
A complete description of the special reflecting device is given by Tate. A brief summary will suffice to indicate the scheme by which photometric measurements at various angles of incidence were made. Fig. I shows a plan of the Brace spectrophotometer and the reflecting system. Fig. 2 shows an elevation of the reflecting system alone. The light source was a flat acetylene flame. Light from a very small area of this flame was focused by lenses upon the collimator slits $S_{1}$ and $S_{2}$ (Fig. I). The mirror surface $M$ was mounted on the center of the table of the reflecting system. Light from the source, after passing through the lens, struck first the fixed prism $A$ (Fig. 2), was successively reflected by

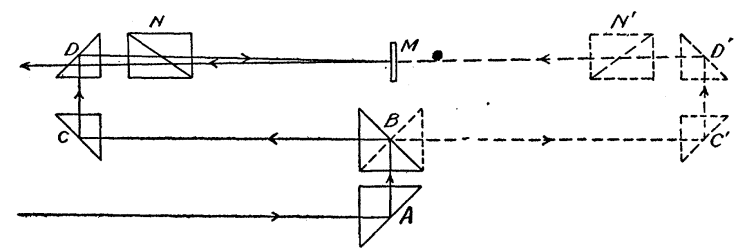

Fig. 2.

prisms $A, B, C$ and $D$, and the mirror $M$-then fell on the slit $S_{2}$ (Fig. 2). The light was plane polarized by passing through the nicol $N$ after reflection from the prism $D$. The prisms $B, C$ and $D$ were fixed relatively to each other and rotated about the axis $A B$ of the system. Light was first directed into the slit without the mirror-along the path $B C^{\prime} D^{\prime}$ - then by reflection from the mirror- $B C D$. This system insured equality of optical path for all angles of incidence on the mirror and also without it. Both the comparison and the test fields of view in the observing telescope were illuminated by the same small area of the flame, so that any variation of intensity of the source affected both fields alike, hence no error could be produced thereby. When the apparatus was in proper adjustment, no fluctuations in intensity could be observed in the field of view. Measurements of relative intensity were made in the usual way by means of the graduated sectors and the adjustable slits $S_{1}$ and $S_{2}$. It is essential that these slit widths be very nearly the same.

The reflection coefficients obtained by means of this apparatus had a probable error of $\mathrm{I}$ to 2 per cent. Determinations of reflection coefficients by means of the polarimeter were made with about ten times this accuracy.

Formula.-The theory of metallic reflection gives the following equations for calculating the refractive indices and the absorption coefficeints:

$$
m_{\theta}=-\frac{\sin \theta \operatorname{tg} \theta \cos 2 \psi \cos 2 \gamma}{\mathrm{I}-\cos 2 \psi \sin 2 \gamma},
$$




$$
\begin{aligned}
m_{\theta} \kappa_{\theta} & =\frac{\sin \theta \operatorname{tg} \theta \sin 2 \psi}{\mathrm{I}-\cos 2 \psi \sin 2 \gamma}, \\
\kappa_{\theta} & =-\frac{\operatorname{tg} 2 \psi}{\cos 2 \gamma},
\end{aligned}
$$

in which $m_{\theta}$ is defined by the equation $m_{\theta}=\nu_{\theta} \cos \rho$, where $\nu_{\theta}$ is the refractive index of the medium at the angle of refraction $\rho$ and the corresponding angle of incidence $\theta$. The quantity $2 \psi$ is a measure of the ellipticity of the reflected light defined by the relation,

$$
E=\operatorname{tg} \psi,
$$

where $E$ is the ratio of the minor to the major axis of the ellipse. The quantity $\gamma$ is the azimuth of the major axis of the elliptic vibration measured with the plane of incidence as the plane of reference. The quantity $\kappa_{\theta}$ is the absorption coefficient of the medium for the angle of incidence $\theta$ such that $e^{2 \pi \kappa_{\theta}}$ gives the ratio of the amplitudes of the vibration at two successive points of equal phase on any normal to the planes of equal amplitude. When light is incident in a transparent medium upon a plane metal mirror, the planes of equal amplitude are parallel to the boundary surface.

The relations between $m_{\theta}, m_{\theta} \kappa_{\theta}$ and $\kappa_{\theta}$ for the angle of incidence $\theta$ and the corresponding constants of the medium at normal incidence are given by Ketteler's equations:

$$
\begin{aligned}
& 2 \nu_{0}^{2}=\sqrt{\left(m_{\theta}^{2}-m_{\theta}^{2} \kappa_{\theta}^{2}+\sin ^{2} \theta\right)^{2}+4 m_{\theta}^{4} \kappa_{\theta}^{2}}+\left(m_{\theta}^{2}-m_{\theta}^{2} \kappa_{\theta}^{2}+\sin ^{2} \theta\right), \\
& 2 \nu_{0}^{2} \kappa_{0}^{2}=\sqrt{\left(m_{\theta}{ }^{2}-m_{\theta}{ }^{2} \kappa_{\theta}{ }^{2}+\sin ^{2} \theta\right)^{2}+4 m_{\theta}{ }^{4} \kappa_{\theta}{ }^{2}}-\left(m_{\theta}{ }^{2}-m_{\theta}{ }^{2} \kappa_{\theta}{ }^{2}+\sin ^{2} \theta\right) \text {, } \\
& 2 m_{\theta}^{2}=\sqrt{\left(\nu_{0}^{2}-\nu_{0}^{2} \kappa_{0}^{2}-\sin ^{2} \theta\right)^{2}+4 \nu_{0}^{4} \kappa_{0}^{2}}+\left(\nu_{0}^{2}-\nu_{0}^{2} \kappa_{0}^{2}-\sin ^{2} \theta\right), \\
& 2 m_{\theta}^{2} \kappa_{\theta}^{2}=\sqrt{\left(\nu_{0}^{2}-\nu_{0}^{2} \kappa_{0}^{2}-\sin ^{2} \theta\right)^{2}+4 \nu_{0}^{4} \kappa_{0}^{2}}-\left(\nu_{0}^{2}-\nu_{0}^{2} \kappa_{0}^{2}-\sin ^{2} \theta\right) \text {. }
\end{aligned}
$$

For convenience in computation, these may be expanded into the following series formulæ, which for all cases used below were accurate to within 0.05 per cent.

$$
\begin{aligned}
& \nu_{0}=m_{0}\left[\mathrm{I}+\frac{\sin ^{2} \theta}{2 m_{\theta}^{2}\left(\mathrm{I}+\kappa_{\theta}^{2}\right)}\right. \\
& \left.\quad+\frac{\mathrm{I}}{2}\left(\frac{m_{\theta}^{2} \kappa_{\theta}^{2}}{m_{\theta}^{2}\left(\mathrm{I}+\kappa_{\theta}^{2}\right)}-\frac{\mathrm{I}}{4}\right) \frac{\sin ^{4} \theta}{m^{4}\left(\mathrm{I}+\kappa_{\theta}^{2}\right)^{2}}-\cdots\right], \\
& \nu_{0} \kappa_{0}=m_{\theta} \kappa_{\theta}\left[\mathrm{I}-\frac{\sin ^{2} \theta}{2 m_{\theta}^{2}\left(\mathrm{I}+\kappa_{\theta}^{2}\right)}\right. \\
& \left.\quad+\frac{\mathrm{I}}{2}\left(\frac{m_{\theta}^{2}}{m_{\theta}{ }^{2}\left(\mathrm{I}+\kappa_{\theta}^{2}\right)}-\frac{\mathrm{I}}{4}\right) \frac{\sin ^{4} \theta}{m_{\theta}^{4}\left(\mathrm{I}+\kappa_{\theta}^{2}\right)^{2}}-\cdots\right] .
\end{aligned}
$$




$$
\begin{aligned}
m_{\theta}=\nu_{0}\left[\mathrm{I}-\frac{\sin ^{2} \theta}{2 \nu_{0}^{2}(\mathrm{I}}+\kappa_{0}^{2}\right) \\
\left.\quad+\frac{\mathrm{I}}{2}\left(\frac{\nu_{0}^{2} \kappa_{0}^{2}}{\nu_{0}^{2}\left(\mathrm{I}+\kappa_{0}^{2}\right)}-\frac{\mathrm{I}}{4}\right) \frac{\sin ^{4} \theta}{\nu_{0}^{4}\left(\mathrm{I}+\kappa_{0}^{2}\right)^{2}}-\cdots\right], \\
m_{\theta} \kappa_{\theta}=\nu_{0} \kappa_{0}\left[\mathrm{I}+\frac{\sin ^{2} \theta}{2 \nu_{0}^{2}\left(\mathrm{I}+\kappa_{0}^{2}\right)}\right. \\
\left.\quad+\frac{\mathrm{I}}{2}\left(\frac{\nu_{0}^{2}}{\nu_{0}^{2}\left(\mathrm{I}+\kappa_{0}^{2}\right)}-\frac{\mathrm{I}}{4}\right) \frac{\sin ^{4} \theta}{\nu_{0}^{4}\left(\mathrm{I}+\kappa_{0}^{2}\right)^{2}}-\cdots\right] .
\end{aligned}
$$

The principal incidence $\bar{\theta}$ and the principle azimuth $\psi$ were calculated by the following approximation formulæ:

$$
\begin{aligned}
&\left.\sin \bar{\theta} \operatorname{tg} \bar{\theta}=\sqrt{\nu_{0}^{2}(\mathrm{I}}+\kappa_{0}^{2}\right) {\left[\mathrm{I}-\frac{\mathrm{I}}{2} \frac{\nu_{0}^{2}\left(\mathrm{I}-\kappa_{0}^{2}\right)}{\nu_{0}^{2}\left(\mathrm{I}+\kappa_{0}^{2}\right)} \cdot \frac{\sin ^{2} \bar{\theta}}{\nu_{0}^{2}\left(\mathrm{I}+\kappa_{0}^{2}\right)}\right.} \\
&\left.+\frac{\mathrm{I}}{8}\left(2-3\left(\frac{\mathrm{I}-\kappa_{0}^{2}}{\mathrm{I}+\kappa_{0}^{2}}\right)^{2}\right) \frac{\sin ^{4} \bar{\theta}}{\nu_{0}^{4}\left(\mathrm{I}+\kappa_{0}^{2}\right)^{2}}-\cdots\right], \\
& \operatorname{tg} 2 \psi=\kappa_{0}\left[\mathrm{I}+\frac{\sin ^{2} \bar{\theta}}{\nu_{0}^{2}\left(\mathrm{I}+\kappa_{0}^{2}\right)}+\frac{\nu_{0}^{2}}{\nu_{0}^{2}\left(\mathrm{I}+\kappa_{0}^{2}\right)} \cdot \frac{\sin ^{4} \bar{\theta}}{\nu_{0}^{4}\left(\mathrm{I}+\kappa_{0}^{2}\right)^{2}}\right. \\
&+\cdots] .
\end{aligned}
$$

The reflection coefficient for the component of the electric vibration perpendicular to the plane of incidence is

$$
J_{s}=\frac{\left(m_{\theta}-\cos \theta\right)^{2}+m_{\theta}^{2} \kappa_{\theta}^{2}}{\left(m_{\theta}+\cos \theta\right)^{2}+m_{\theta}^{2} \kappa_{\theta}^{2}}
$$

for the component of the electric vibration in the plane of incidence,

$$
J_{p}=J_{s} \frac{\left(m_{\theta}-\sin \theta \operatorname{tg} \theta\right)^{2}+m_{\theta}^{2} \kappa_{\theta}^{2}}{\left(m_{\theta}+\sin \theta \operatorname{tg} \theta\right)^{2}+m_{\theta}^{2} \kappa_{\theta}^{2}} .
$$

For normal incidence $(\theta=0)$ both these equations reduce to the standard form of the reflection coefficient at normal incidence, namely

$$
J_{0}=\frac{\nu_{0}^{2}\left(\mathrm{I}+\kappa_{0}^{2}\right)+\mathrm{I}-2 \nu_{0}}{\nu_{0}^{2}\left(\mathrm{I}+\kappa_{0}^{2}\right)+\mathrm{I}+2 \nu_{0}} .
$$

\section{Experimental Results.}

Steel.-Observations were made on a steel mirror taken from a König tuning fork. This mirror which had an excellent plane surface was thoroughly cleaned, then rubbed slightly with chamois and rouge before the observations were made. On account of the small ellipticity produced at small angles of incidence, and the difficulty of measuring such 
ellipticities accurately, the smallest angle of incidence used was $40^{\circ}$. Table I. gives the results obtained, for the wave-length $580 \mu \mu$ showing the magnitude of the reflection coefficients calculated from the polarimetric measurements (columns 7 and 9), and those directly observed with the spectrophotometer (columns 8 and Io). It is seen that the most consistent values for $\nu_{0}, \nu_{0} \kappa_{0}$ and $\kappa_{0}$ are given for angles of incidence of $40^{\circ}, 50^{\circ}, 60^{\circ}$ and $70^{\circ}$. These constants were calculated from the corresponding $m_{\theta}, m_{\theta} \kappa_{\theta}$ and $\kappa_{\theta}$ by equations (4). The average values of $\nu_{0}, \nu_{0} \kappa_{0}$ and $\kappa_{0}$ for these angles were taken as the most probable and given in the table under the incidence angle o. The values of $m_{\theta}, m_{\theta} \kappa_{\theta}$ and $\kappa_{\theta}$ for the angles of incidence $10^{\circ}, 20^{\circ}$, and $30^{\circ}$ were calculated from the average $\nu_{0}, \nu_{0} \kappa_{0}$ and $\kappa_{0}$ by equations (5).

TABLE I.

Steel Mirror, $\lambda=580 \mu \mu$.

\begin{tabular}{l|c|c|c|c|c|c|c|c|c|c|c|c}
\hline \multicolumn{1}{c|}{$\boldsymbol{2} \gamma$} & $\mathbf{2} \psi$ & $m_{\theta}$ & $\kappa_{\theta}$ & $m_{\theta^{\kappa}}$ & $\begin{array}{c}J_{s} \\
\text { Calc. }\end{array}$ & $\begin{array}{c}J_{s} \\
\text { Obs. }\end{array}$ & $\begin{array}{c}J_{p} \\
\text { Calc. }\end{array}$ & $\begin{array}{c}J_{p} \\
\text { Obs. }\end{array}$ & $v_{0}$ & $\kappa_{0}$ & $\nu_{0} \kappa_{0}$ \\
\hline 0 & & & 2.360 & 1.360 & 3.214 & .560 & .555 & .563 & .558 & & & \\
\hline 10 & & & 2.358 & 1.365 & 3.217 & .569 & .555 & .558 & .547 & & & \\
20 & & & 2.351 & 1.372 & 3.226 & .578 & .573 & .536 & .541 & & & \\
30 & 0 & & 2.345 & 1.382 & 3.239 & .609 & .599 & .514 & .509 & & & \\
40 & 99.08 & 12.42 & 2.337 & 1.395 & 3.259 & .645 & .634 & .473 & .470 & 2.367 & 1.359 & 3.217 \\
50 & 105.48 & 20.67 & 2.320 & 1.413 & 3.278 & .677 & .685 & .407 & .408 & 2.363 & 1.362 & 3.218 \\
60 & 116.54 & 32.55 & 2.298 & 1.429 & 3.283 & .753 & .746 & .342 & .340 & 2.353 & 1.363 & 3.207 \\
70 & 141.26 & 48.30 & 2.294 & 1.439 & 3.302 & .825 & .811 & .261 & .262 & 2.359 & 1.362 & 3.212 \\
75 & 169.04 & 55.08 & 2.273 & 1.459 & 3.318 & .863 & .869 & .242 & .240 & 2.341 & 1.377 & 3.222 \\
80 & 211.25 & 51.28 & 2.255 & 1.459 & 3.290 & .905 & .895 & .277 & .270 & 2.326 & 1.372 & 3.190 \\
82.5 & 230.65 & 42.85 & 2.234 & 1.463 & 3.269 & .927 & .930 & .339 & .338 & 2.306 & 1.374 & 3.167 \\
85 & 245.82 & 30.78 & 2.247 & 1.454 & 3.267 & .957 & 1 & .456 & 1 & 2.320 & 1.365 & 3.165 \\
\hline \hline
\end{tabular}

$\bar{\theta}=76^{\circ} .42 \psi=27^{\circ} .63$

Fig. 3 shows the curves in which reflection coefficients are plotted against angles of incidence. The clear circles represent values calculated from polarimetric measurements; the black circles represent values observed upon the spectrophotometer.

It is seen that the observed and calculated values of the reflection coefficients agree within the errors of measurement. The average difference between calculated and observed values is I per cent. and the maximum difference is 2.5 per cent. It will be noticed, however, that the values of $\nu_{0}$ and $\nu_{0} \kappa_{0}$ given by polarimetric observations at different angles of incidence do not agree within the errors of measurements; there is a marked decrease in the values of $\nu_{0}$ and $\nu_{0} \kappa_{0}$ for the larger

${ }^{1}$ Photometric observations on the mirror at this incidence could not be obtained on account of the small effective aperture of the mirror. 
angles of incidence. At $\theta=85^{\circ}$, the value of $\nu_{0}$ is $\mathrm{I} .7$ per cent. less than the accepted value for that constant (mean of four best values), and $\nu_{0} \kappa_{0}$ is I. 6 per cent. less than the accepted value, while the instrumental determination of any one of these quantities is correct to within

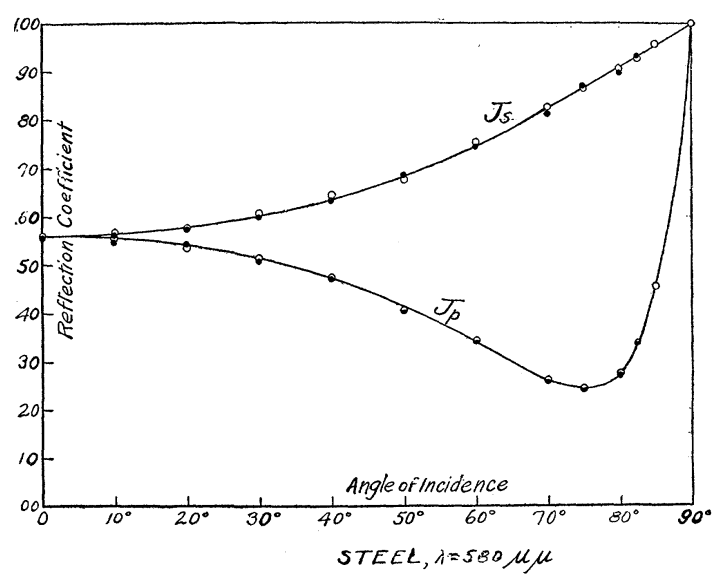

Fig. 3.

.I per cent. It follows then that the theory (Ketteler's equations) giving the relations between the various refractive indices and absorption coefficients for the different angles of incidence does not hold within the

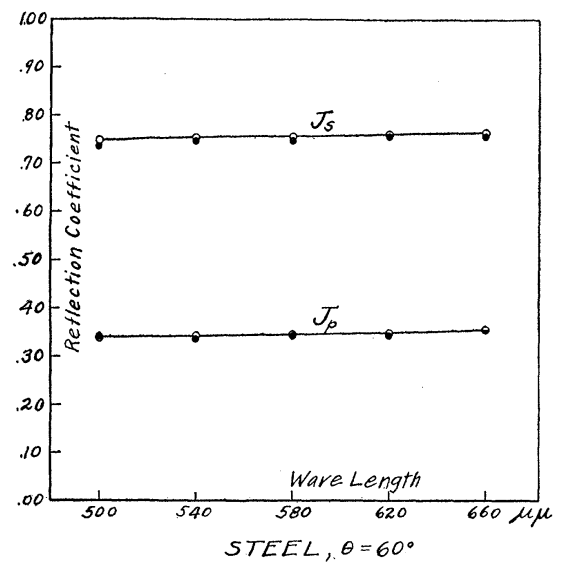

Fig. 4.

errors of measurement especially for angles of incidence larger than $70^{\circ}$. This discrepancy is also indicated by the fact that some of the calculated reflection coefficients lie farther from the smooth curve than the error in their determination would allow. This must arise, of course, from 
imperfections in the mirror. It seems quite likely that polarimetric measurements on the mirror at any angle of incidence should give correct reflection coefficients for that angle of incidence; but the calculation of the constants for other angles of incidence (using Ketteler's equations) might not give correct values - the errors being especially large for large angle of incidence. However, the observations show that the theory holds very well for mirrors as perfect as we were able to produce.

Table II. gives the results obtained from the same mirror by keeping

TABLE II.

Steel Mirror, $\theta=60^{\circ}$.

\begin{tabular}{c|c|c|c|c|c|c|c|c|c}
\hline$\lambda$ & ${ }^{2 \gamma}$ & $2 \psi$ & $m_{\theta}$ & $\kappa_{\theta}$ & $m_{\theta} \kappa_{\theta}$ & $\begin{array}{c}J_{\delta} \\
\text { Calc. }\end{array}$ & $\begin{array}{c}J \\
\text { Obs. }\end{array}$ & $\begin{array}{c}J_{p} \\
\text { Calc. }\end{array}$ & $\begin{array}{c}J_{p} \\
\text { obs. }\end{array}$ \\
\hline 500 & 117.47 & 35.50 & 2.030 & 1.543 & 3.132 & .749 & .736 & .339 & .341 \\
540 & 116.83 & 33.85 & 2.171 & 1.487 & 3.226 & .751 & .744 & .342 & .337 \\
580 & 116.25 & 32.47 & 2.301 & 1.439 & 3.308 & .754 & .746 & .344 & .342 \\
620 & 115.64 & 31.47 & 2.396 & 1.415 & 3.389 & .759 & .754 & .349 & .343 \\
660 & 114.98 & 30.60 & 2.482 & 1.400 & 3.475 & .762 & .755 & .356 & .356 \\
\hline
\end{tabular}

the angle of incidence constant and varying the wave-length of the incident light. The angle chosen was $60^{\circ}$ and observations were made at five points in the spectrum. The observations were taken about three weeks after those of Table I. The mirror was slightly repolished before the observations were made. Fig. 4 gives the curves obtained by plotting these reflection coefficients against wave-lengths. These data show good agreement between the calculated and observed values.

TABLE III.

Gold, $\lambda=620 \mu \mu$.

\begin{tabular}{|c|c|c|c|c|c|c|c|c|c|c|c|c|}
\hline$\theta$ & $2 \gamma$ & $2 \psi$ & $m_{\theta}$ & $\kappa_{\theta}$ & $m_{\theta^{\kappa}} \theta$ & $\underset{\text { Calc. }}{J_{s}}$ & $\begin{array}{c}J_{s} \\
\text { Obs. }\end{array}$ & $\begin{array}{c}J_{p} \\
\text { Calc. }\end{array}$ & $\begin{array}{c}J_{p} \\
\text { Obs. }\end{array}$ & $\nu_{0}$ & $\kappa_{0}$ & $\nu_{0} \kappa_{0}$ \\
\hline 0 & & & .3104 & 10.03 & 3.108 & .891 & .880 & .891 & .883 & & & \\
\hline 20 & $\circ$ & o & .3086 & 10.15 & 3.126 & .898 & .884 & .884 & .877 & & & \\
\hline 40 & 91.95 & 19.15 & .3104 & 10.20 & 3.167 & .916 & .912 & .859 & .862 & .3169 & 9.79 & 3.102 \\
\hline 60 & 96.17 & 49.37 & .2977 & 10.85 & 3.228 & .943 & .942 & .819 & .815 & .3089 & 10.07 & 3.109 \\
\hline 70 & 111.23 & 75.73 & .2993 & 10.86 & 3.248 & .963 & .972 & .805 & .804 & .3125 & 9.96 & 3.108 \\
\hline 80 & 260.83 & 60.37 & .2956 & 11.04 & 3.262 & .980 & .987 & .836 & .828 & .3098 & 10.04 & 3.108 \\
\hline 85 & $267.00 \mid$ & 31.52 & .2744 & 11.72 & 3.215 & .989 & .978 & .905 & .901 & .2884 & 10.60 & 3.055 \\
\hline
\end{tabular}

Gold.-A gold mirror was made by electroplating with gold cyanide upon polished speculum metal and afterwards polishing the plated surface with chamois skin and rouge. Several mirrors were made by 
TABLE IV.

Gold, $\theta=60^{\circ}$.

\begin{tabular}{|c|c|c|c|c|c|c|c|c|c|}
\hline$\lambda$ & $2 \gamma$ & $2 \psi$ & $m_{\theta}$ & $\kappa_{\theta}$ & $m_{\theta^{\kappa} \theta}$ & $\begin{array}{c}J_{s} \\
\text { Calc. }\end{array}$ & Obs. & $\begin{array}{c}J_{p} \\
\text { Calc. }\end{array}$ & $\begin{array}{c}J_{p} \\
\text { Obs. }\end{array}$ \\
\hline 500 & $138^{\circ} .92$ & $59^{\circ} .89$ & .8461 & 2.287 & 1.936 & .717 & .683 & .314 & .332 \\
\hline 540 & 112.3 & 6 & .49 & 4.8 & 2.364 & .849 & .832 & .588 & .595 \\
\hline 580 & 100.33 & 54 & .35 & 7.9 & 2.831 & 918 & .908 & .746 & .743 \\
\hline 620 & 96.30 & 49.45 & .3024 & 10.65 & 3.221 & .945 & .942 & .818 & .818 \\
\hline 660 & 94.50 & 45.22 & .2783 & 12.84 & 3.575 & .960 & .943 & .859 & .848 \\
\hline $\left.\begin{array}{rl}\theta & =80^{\circ} \\
\lambda & =540\end{array}\right\}$ & 258.92 & 45.72 & .4448 & 5.334 & 2.373 & .949 & .964 & .723 & .720 \\
\hline $\begin{array}{l}\theta=50^{\circ} \\
\lambda=580\end{array}$ & 95.30 & 36.01 & .3506 & 7.870 & 2.759 & .896 & .891 & .772 & .769 \\
\hline
\end{tabular}

this method and for the observations one was chosen which had a very brilliant and smooth surface. Measurements were made at a wavelength of $620 \mu \mu$, varying the angle of incidence; and, at an angle of incidence of $60^{\circ}$, varying the wave-length. These results are given in Tables III. and IV., and are shown graphically in Figs. 5 and 6 . The

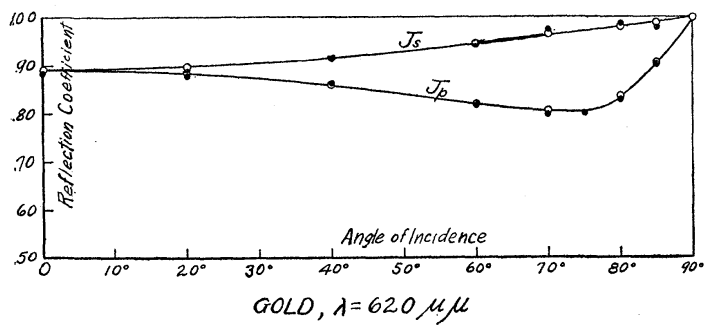

Fig. 5.

average difference between observed and calculated reflection coefficients is I.I per cent. and the maximum is 5.7 per cent. This latter exceptionally poor agreement occurs for the two reflection coefficients at $\theta=60^{\circ}, \lambda=500 \mu \mu$, values that lie on the steepest portions of the curves. Omitting these two values, the average difference between observed and calculated magnitudes is .8 per cent. and the maximum I. 8 per cent. Similar to the case of the steel mirror the most consistent values of $\nu_{0}$ and $\nu_{0} \kappa_{0}$ are given at angles of $60^{\circ}, 70^{\circ}$ and $80^{\circ}$, while those for $85^{\circ}$ are low. This seems to indicate that imperfections in the surface have greater effect when viewed at the largest angles of incidence, where the roughness or pits in the surface would of course make up a larger part of the visual area.

The smallest angle of incidence at which reliable polarimetric observations could be made was $40^{\circ}$. The values of the constants for $0^{\circ}$ and $20^{\circ}$ 
were calculated therefore from observations at $60^{\circ}, 70^{\circ}$ and $80^{\circ}$ by equations (4), (5), (6) and (7).

It will be noticed that in the cases of both steel and gold, the minimum of $J_{p}$ is very nearly at the principal angle $\bar{\theta}$. In fact, the principal angle is at the maximum of the ratio $J_{s} / J_{p}$, which is the same as saying that

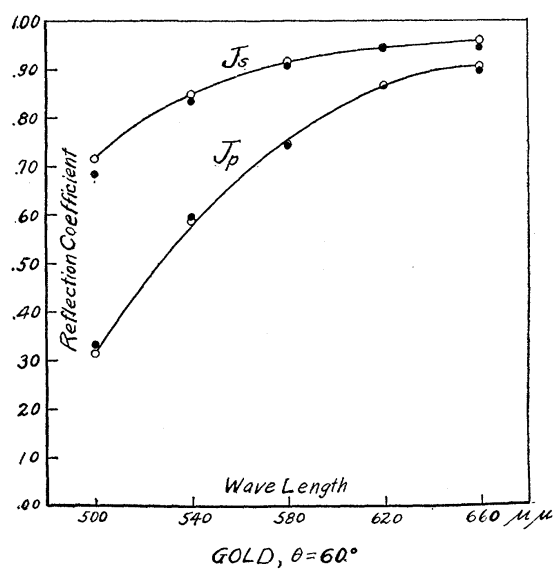

Fig. 6.

the principal azimuth is the minimum azimuth of restored polarization. These considerations follow from the theory.

To illustrate the effect of a visibly imperfect surface upon the agreement of observed and calculated reflection coefficients, results are given in Table V. from observations made upon a gold mirror which had a

TABLE V.

Imperfect Gold Mirror, $\lambda=620 \mu \mu$.

\begin{tabular}{|c|c|c|c|c|c|c|c|c|c|}
\hline$\theta$ & $2 \gamma$ & $2 \psi$ & $m_{\theta}$ & $\kappa_{\theta}$ & $m_{\theta^{\kappa} \theta}$ & $\begin{array}{c}J_{s} \\
\text { Calc. }\end{array}$ & $\begin{array}{c}J_{s} \\
\text { Obs. }\end{array}$ & $\begin{array}{c}J_{p} \\
\text { Calc. }\end{array}$ & $\begin{array}{l}J_{p} \\
\text { Obs. }\end{array}$ \\
\hline $60^{\circ}$ & $9 \stackrel{0}{9} .31$ & 51.32 & .2728 & 11.338 & 3.094 & .946 & .872 & .824 & .772 \\
\hline 80 & 261.72 & 57.33 & .2831 & 10.826 & 3.064 & .979 & .837 & .838 & .817 \\
\hline 0 & & & .2841 & 10.457 & 2.972 & .880 & .806 & .880 & .817 \\
\hline
\end{tabular}

very slight "grayness" in its surface. This grayness could be seen when the surface was viewed at the proper angle. The observed values are from 2 to 8 per cent. too low. Doubtless the chief cause of this discrepancy is that any fine scratches or pits in the surface decrease the quantity of regularly reflected light-giving diffuse reflection-without appreciably affecting the ellipticity of the light which is regularly reflected. 


\section{ConCLusions.}

The reflection coefficients of a steel mirror and of a gold mirror for the two components of polarized light for various angles of incidence and for various wave-lengths were determined practically simultaneously by the indirect polarimetric method and the direct photometric method. The results agree on the average within one per cent., the observed values lying both above and below the calculated values. The results show, therefore, that the theory by which reflection coefficients of metals are calculated from polarimetric measurements holds very closely for the mirrors tested. The polarimetric method gives values which can be duplicated to within .I to .2 per cent.; whereas the uncertainty of the photometric method is about 2 per cent. The polarimetric method for determining the reflection coefficient of a mirror is valid however only for mirrors having a very perfect surface.

Ketteler's equations, which give the variation of the refractive index and of the absorption coefficient with angle of incidence, hold very well except for the largest angles of incidence. There the deviation amounts to from 2 to 7 per cent. in the refractive indices and absorption coefficients. The fact that some of the polarimetrically determined reflection coefficients do not lie upon a smooth curve indicates slight deviations from Ketteler's equations.

In conclusion, the writer wishes to thank Professor Skinner for suggesting the problem and directing the investigation. He is also indebted to Professor Tuckerman and Drs. Tool and Tate for many helpful suggestions.

The University of Nebraska, Brace Laboratory of Physics. 